\title{
Periodontal Osseous Defects: A Review
}

\author{
${ }^{1}$ Bharath Chandra GNR, ${ }^{2} \mathrm{KL}$ Vandana
}

\begin{abstract}
Periodontitis is an inflammatory process affecting the periodontal tissues caused by multi-factorial origin. Among all the characteristic signs of periodontal disease, loss of support from alveolar boneis the one which usually represents theanatomical sequela to the progression of periodontitis apically.The bone loss which is induced by periodontitis occur either single or in different combination forms. The identification of these osseous defects on surgical exposure of bone is clinically challenging as the osseous and it becomes imperative for a clinician to understand these defects and categorize them well to have better therapeutic approaches. Intimate knowledge of all these periodontal osseous defects associated with periodontal disease is essential. So this review is aimed at classification and deep insight which will be helpful for proper diagnosis and treatment of periodontal osseous defects.
\end{abstract}

Keywords: Alveolar process, Bone resorption, Periodontitis.

How to cite this article: Chandra GNRB, Vandana KL. Periodontal Osseous Defects: A Review. CODS J Dent 2017;9(1):22-29.

Source of support: Nil

Conflict of interest: None

\section{INTRODUCTION}

Periodontium is composed of both soft and hard tissues in which alveolar bone is the part which forms and also supports the teeth in both maxilla and mandible. Alveolar bone formation occurs as the eruption of tooth takes place in order to facilitate the osseous attachments to the periodontal ligament and disappears once the tooth is lost. ${ }^{1}$

Alveolar bone has its embryological origin from the initial condensation of ecto-mesenchyme around the early tooth germ. The alveolar process house the teeth and exist as long as teeth are present in it. The sharpey's fibres are embedded in the alveolar bone proper which is the compact bone, compromised of oral and buccal cortical plates and the cancellous bone located between them. ${ }^{1}$

\footnotetext{
${ }^{1}$ Senior Lecturer, ${ }^{2}$ Senior Professor

${ }^{1}$ Department of Periodontics, Panineeya Mahavidyalaya Institute of Dental Sciences, Hyderabad, Telangana, India

${ }^{2}$ Department of Periodontics, College of Dental Sciences, Davangere, Karnataka, India

Corresponding Author: KL Vandana, Senior Professor, Department of Periodontics, College of Dental Sciences, Davangere, Karnataka, India, Phone: +919448393364, e-mail: vanrajs@gmail.com
}

The bone loss in periodontal disease occurs at local sites, but it is regulated by both systemic and local factors. Bone resorption is probably the most critical factor in periodontal attachment loss leading to eventual tooth loss. ${ }^{2}$ Radiographically it is diagnosed by evidence of "bone loss" around the tooth. Normally the crest of alveolar bone is situated between 0.4 to $1.97 \mathrm{~mm}$ approximately apically to the cemento-enamel junction (CEJ) of thatparticular tooth. ${ }^{3}$

The bone loss which is induced by periodontitis i.e osseous defects occur either single or in different combination forms. The identification of these osseous defects on surgical bone exposure is clinically challenging as the osseous surgeries are based on this diagnosis. It becomes imperative for a clinician to understand these defects and categorize them well to have better periodontic therapeutic approaches.

Periodontal osseous defects (POD) is an important clinical reality, however, it's classification and description are not being dealt in regular universally accepted text books. Hence, an attempt is made in this review paper to revisit, modify and describe various aspects of POD for the first time in literature.

\section{Incidence and Prevalence}

The changes which are observed in the alveolar process architecture may differ in form, distribution and degree within same individual at different sites as well as between individuals.

The prevalence of vertical defect was higher in male patients (14.95\%) when compared to female patients (8.2\%) and also it was rare in patients with dental awareness (de Toledo et al 2012). ${ }^{4}$ Vertical defects are commonly associated with posterior teeth (Baljoon et al.), ${ }^{5}$ with the higher prevalence in mandibular posterior teeth (33.8\%) (Vrotsos et al., Kasaj et al.). ${ }^{6,7}$

Vertical defects are commonly associated with molars with higher prevalence of crater formation (26.5\%), followed by circumferential defects $(23.4 \%)$ and 3 wall defects (20.08\%) (Wu et al.). ${ }^{8}$

\section{Osseous Defects}

Definition: Osseous defects are defined as the alterations in the morphology of the alveolar bone (GPT). These occur normally (anatomic variations) and disease induced. As these defects act a crucial role either in initiating or 
progressing the disease, alveolar architecture influences the occurrence of disease induced defects. Various classifications for osseous defects are Goldman and Cohen, ${ }^{9}$ Pritchard, ${ }^{10}$ classification by Clarke, ${ }^{11}$ Papapanou and Tonetti ${ }^{12}$ proposed as shown in Table 1 .

Considering the short comings of the existing classifications on osseous defects, by this review an attempt is made to propose a new classification of periodontal osseous defects. The newly proposed classification of POD by Vandana and Bharath ${ }^{13}$ includes the various types of POD categorized under specific heading which is clinically applicable and easy to comprehend which is shown in Flow Chart 1.

\section{Nomenclature of Periodontal Osseous Defects of the Alveolar Process}

\section{Developmental Osseous Defects or Abberations}

The Bulbous Bone Contours: The enlargements in bone which are caused because of exostoses, buttressing bone formation and sometimesas an adaptation to function ${ }^{14}$ (Fig 1).

The Exostoses: Exostoses are out growths of varied shape and size in the bone which can also occur as sharp ridges, nodules either large or small, projections which may be appeared as spikes, or in any one of these combinations. ${ }^{15}$ Fenestrations and Dehiscence: These can be developmental or due to diseases.

\section{Fenestrations}

The areas in which the root is devoid or denuded of supporting alveolar bone and the surface of the root is covered by periosteum of bone, overlying gingiva and in these type of defects we can see the intact marginal bone and generally seen as isolated areas.

Dehiscence: These are the areas with denuded bone where marginal boneis lost. They are seen often on the facial bone compared to lingual and common in the anterior teeth compared top osterior teeth with bilateral frequency. The predisposing factors are prominent contours of roots, malpositions, and protrusion of the root labially combined with covering of thin bony plate (Fig. 2).

These defects are considered to be important as they can play a role in changing or even complicating the outcome of periodontal surgery. ${ }^{2}$ They can occur either in the maxilla or mandible with varied distribution as described earlier in which fenestrations are more commonly show prevanlencein the maxilla approximately $74.679 \%$ while dehiscences are mostly seen in the mandible approximately $71.613 \% .^{16}$

Of all the affected teeth fenestrations are more prevalent in the posterior maxilla regions approximately $49.036 \%$ and dehiscences are more prevalent in the anterior mandibular region approximately $15.631 \%$. The most often affected teeth by fenestration are the maxillary first molar (42.62\%), mandibular first molar (16.02\%) and maxillary first premolar (14.42\%) and the most often

Flow Chart 1: The modified classification of periodontal osseous defects (POD) by Vandana and Bharath ${ }^{13}$

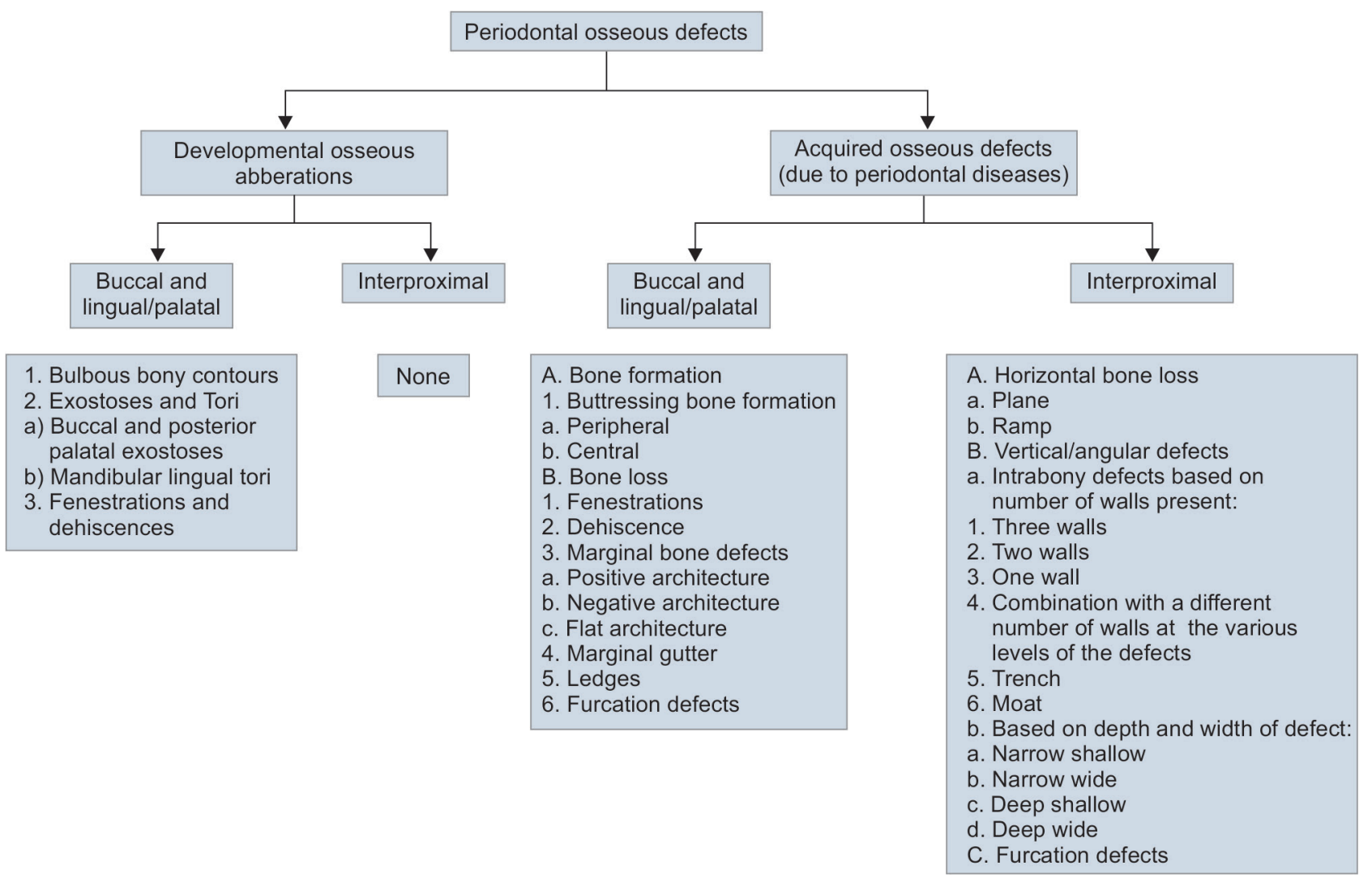


Table 1: Various classifications of osseous defects

\section{Goldman and Cohen ${ }^{9}$}

1. Three osseous walls.

A. Proximal, buccal and lingual walls

B. Buccal, mesial and distal walls

C. Lingual, mesial and distal walls

2. Two osseous walls

A. Buccal and lingual (crater) walls

B. Buccal and proximal walls

C. Lingual and proximal walls

3. One osseous wall

A. Proximal wall

B. Buccal wall

C. Lingual wall

4. Combination

A. Three walls plus two walls

B. Three walls plus two walls plus one wall

Note: This classification concentrates only on disease induced osseous defects which don't include furcation defects. Developmental defects are not included.

Pritchard $1965^{10}$ has classified those osseous defects caused by periodontal disease as interproximal craters,

Inconsistent margins,

Hemisepta,

Furca invasions,

Intrabony defects (infrabony defects with three osseous walls), and combinations of those defects.

These absorptive lesions may also be complicated by anatomic aberrations of the alveolar process, i.e. thick marginal ledges, exotoses, and tori.

Additional anatomic aberrations of the alveolar process are isolated areas in which the root is denuded of bone to a varying extent. If the marginal bone remains intact, such denuded areas are called fenestrations; if such areas involve marginal bone, they are termed dehiscences.

Note: The osseous defects are being just named without a organized structural classification

\section{Classification by Clarke ${ }^{11}$}

A. Vestibular, lingual or palatal structures or defects

- Normal anatomic structures
(a) External oblique ridge
(b) Retromolar triangle
(c) Mylohyoid ridge
(d) Zygomatic process

- Exostoses and tori

(a) Mandibular lingual tori

(b) Buccal and posterior palatal exostoses

- Dehiscence

- Fenestrations

- Reverse osseous architecture
B. Vertical defects

- Three walls

- Two walls

- One wall

- Combination with a different number of walls at the various levels of the defects.

C. Furcation Defects

- Class I or incipient

- Class II or partial

- Class III or through and through.

Note: This classification is relatively better conceptualised, however, still lacks the necessary systematic approach. The disease induced marginal bone architecture such as positive architecture, negative architecture, and flat architecture are missing. The nomenclature such as crater and hemisepta are not included.

Perio2000: Classifications are generally based upon specific morphological criteria and are aimed at guiding clinicians with their diagnosis, treatment and prognosis. A first level of classification differentiates between suprabony defects, infrabony defects, and interradicular or furcation defects. ${ }^{12}$

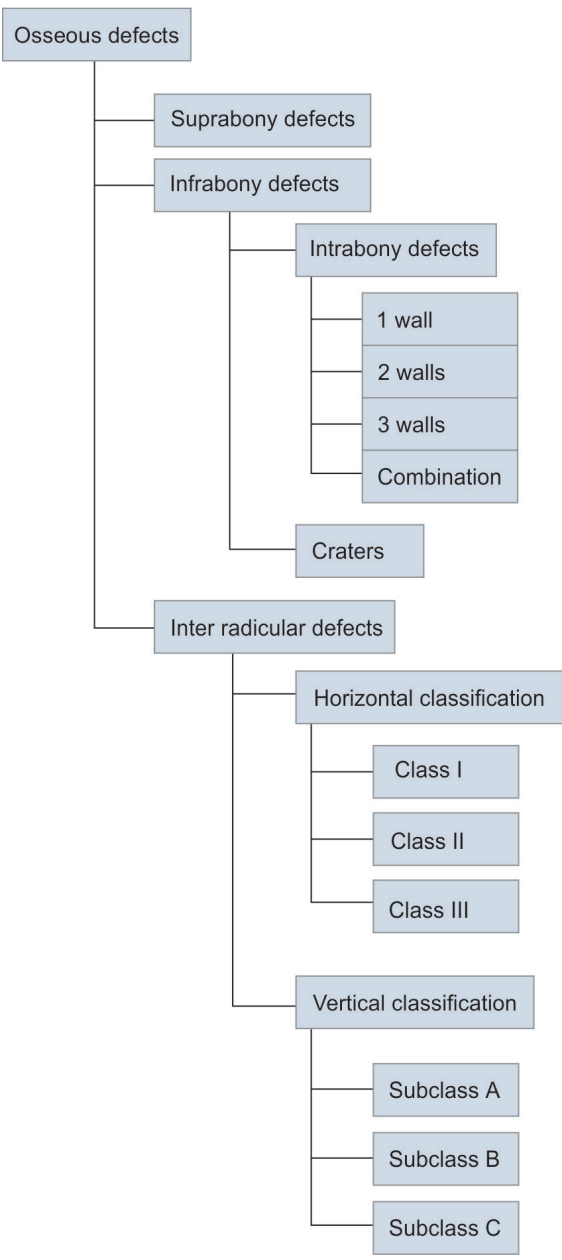

Note: The term supra bony defect is not appropriate or misleading, i.e., no defect exists above the bone. 


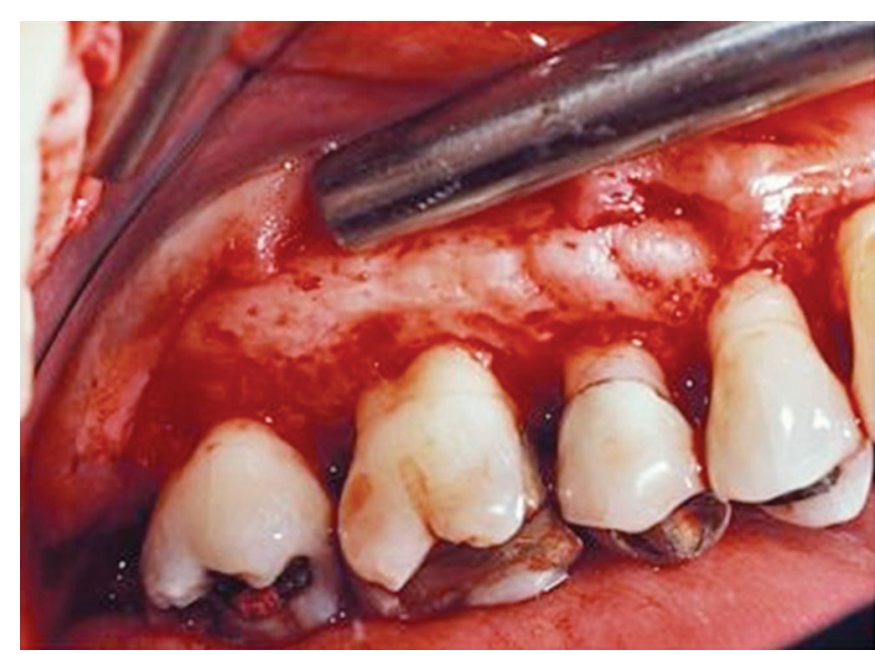

Fig. 1: Bulbous bone contours

affected teeth by dehiscence are the mandibular canine $(40.645 \%)$, mandibular first premolar $(18.06 \%)$ and maxillary canine $(17.41 \%){ }^{16}$

\section{Acquired Osseous Defects}

\section{Alveolar Bone formation}

The Buttressing Bone (Lipping) formation: Alveolar bone formation at some instances occurs to buttress the bony trabeculae which are weakened due to bone resorption and if it occurs within the jaw, termed as central buttressing bone formation and if it occurs on the external surface of bone, termed as peripheral buttressing bone formation which usually causes bulging of the contour of bone, termed as lipping, which at sometimes may accompany the production of osseous craters and angular bone defects. ${ }^{17}$

\section{Alveolar Bone loss}

\section{Buccal and lingual/palatal}

Marginal Defects: The Architecture is a commonly used term in periodontics to describe gingival and/or bony form. ${ }^{2}$ In physiologic architecture concept the bone or soft tissue form includes positive architecture in a vertical dimension, bucco-lingual contours devoid of exostoses, ledges, interradicular grooves and interdental bonecrest is always coronal to the midfacial bone.The marginal bone shows scalloping and festooning the buccal lingual bone presents as tapering margin. ${ }^{2}$

Positive architecture is the marginal bone loss which occurs in such a way that the interdental gingiva or bone crest is coronally located tomargins of their midfacial or midlingual surfaces. Reverse architecture is the marginal bone loss which occurs in such a way that the interdental gingiva or bone crest is apically located to themargins of their midfacial or mid-lingual levels. ${ }^{2}$ These type of defects are produced by loss of interdental bone, including the facial and/or lingual plates without loss of radicular bone and thus the normal architectureis

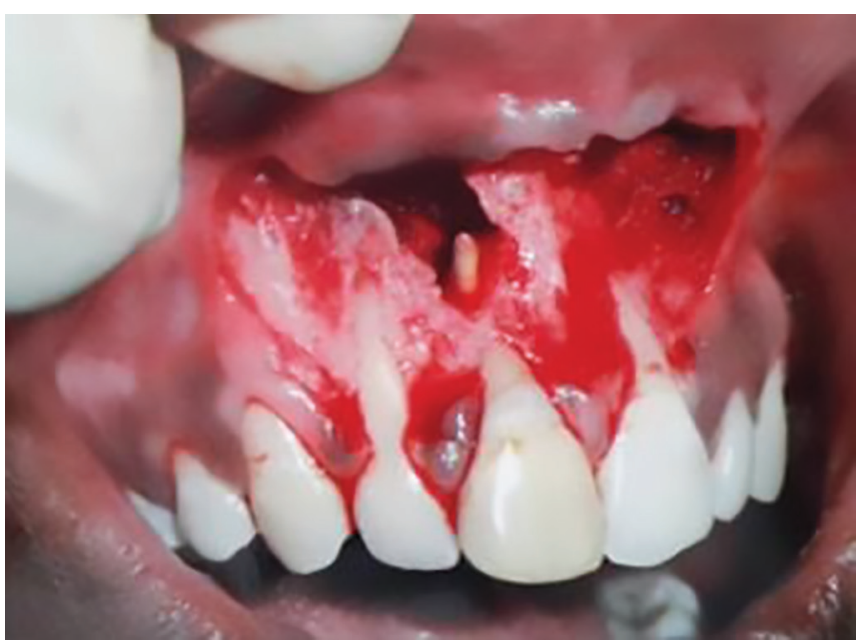

Fig. 2: Pathologic fenestration irt 11, dehiscence irt 12

reversed and these type of defects are most commonly seen in the maxilla ${ }^{2}$ (Fig. 3).

Flat architecture: The marginal bone loss occurs in such a way that both interdenytal crest and midfacial and midlingual margins remain at same level. ${ }^{2}$ Due to periodontal disease, the marginal bone remains thin or thickened either with positive architecture, negative architecture, and flat architecture.these marginal bone alterations can be restricted to either 2 to 3 teeth or whole segment/ quadrant may be involved.

Marginal Gutter: The shallow linear defect which is present in between the marginal alveolar bone of the radical cortical plate or inter-dental alveolar bone $e^{14}$ (Figs 4 and 5).

Ledges : These are plateau-like margins of bone which are caused due to resorption of the thickened bone plates usually the buccal \labial bone ${ }^{2}$ (Fig. 6).

Furcation Involvement: The furcation involvement usually refers to the periodontal disease invasion of bifurcation or trifurcation of a multirooted teeth. The most common sites are usually the mandibular first molars and the involvement of maxillary teeth increase with age. ${ }^{18}$

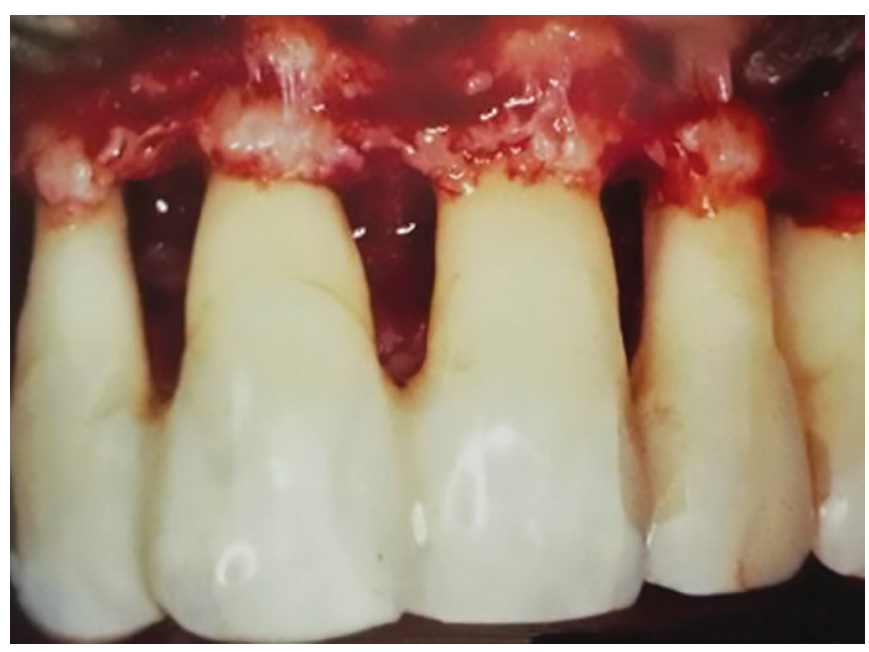

Fig. 3: Reverse architecture irt 12,11,21,22 


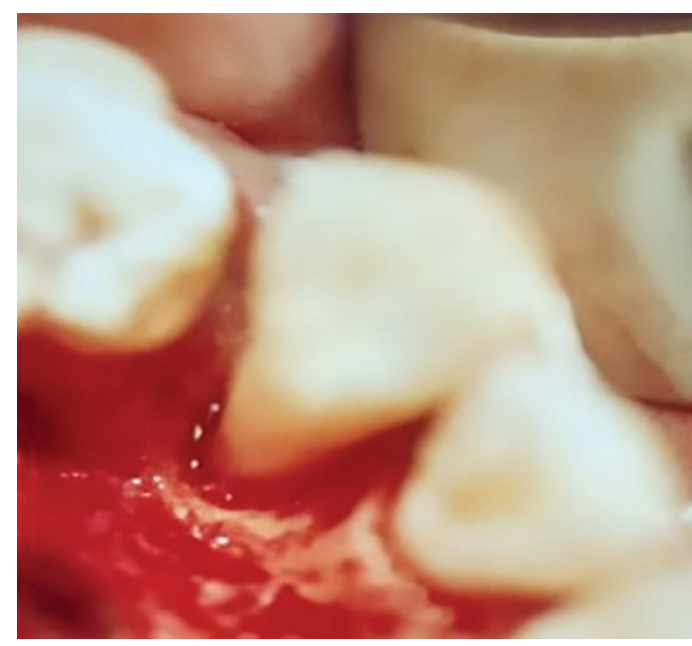

Fig. 4: Gutter irt 13 palatally

The denuded areas of furcation involvemen may be covered by the walls of the pocket or may be visible clinically and the furcation involvement is determined by exploration with a blunt probe usually naber's probe along with a blast of warm air simultaneously in order to facilitate bettervisualization. ${ }^{2}$

Furcation involvement is classified as grades I to IV based on the amount of destruction of tissues. ${ }^{2}$ In grade I lesion is seen as incipient bone loss, in grade II furcation lesion is seen as partial boneloss (cul-de-sac), in grade III the lesion is the total bone with through-and-throug hopening of the furcation is present and in grade IV the lesion is similar to grade III, but the lesion is with gingival recession thus the furcation invovlvmentis exposed and viewed clinically.

The pattern of destruction infurcation involvement varies with the degree of involvement and in different cases and alveolar bone loss around each root may be either horizontal or angular, and frequently a crater may develop in the interradicular region ${ }^{2}$ (Figs 7 and 8).

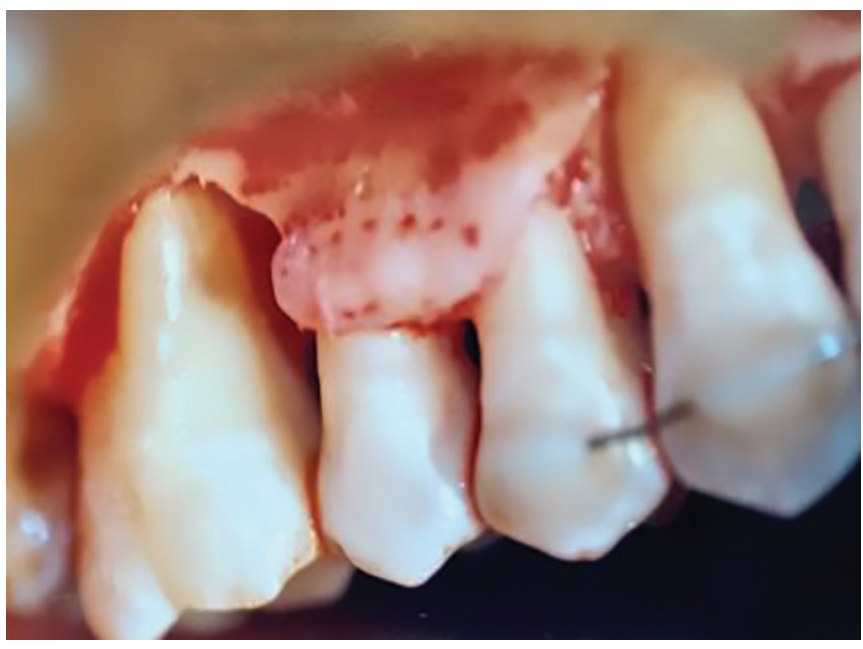

Fig. 6: Ledge irt 15

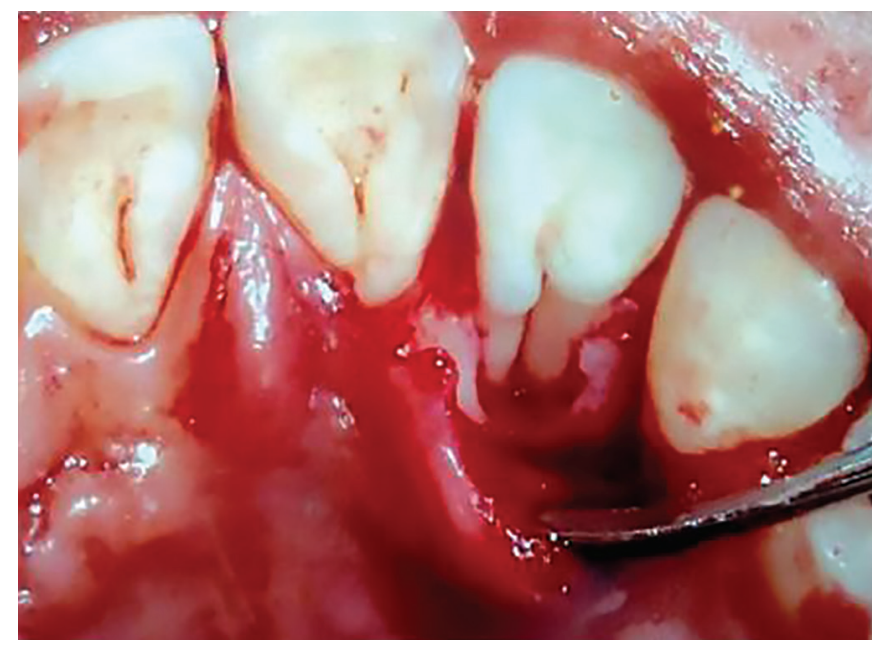

Fig. 5: Mid palatally intrabony defect with palatal groove irt 22

\section{Interproximal Defects}

Horizontal defects: Horizontal pattern of boneloss in periodontal disease is the most common type in which the height of the bone is reduced with the margin of bone roughly remainining perpendicular to surface of tooth and the facial and lingual plates, the interdental septum are also affected but may not be to an equalextent around the same tooth. $^{2}$

Plane: The term plane is applied if both the alveolar bone and supporting bone are lost approximately to the same extentin such a manner that the defect margins are located at same level. ${ }^{19}$

Ramp: The term ramp is applied to deformity which results if both alveolar bone and its supporting bone are lost to the approximately same extent such that the margins of the defect are located at different levels. ${ }^{19}$

Vertical Bone Loss: Alveolar bone loss which occur in an oblique direction and leavesa hollowed out thorough in the bone along the rootis called as vertical or angular

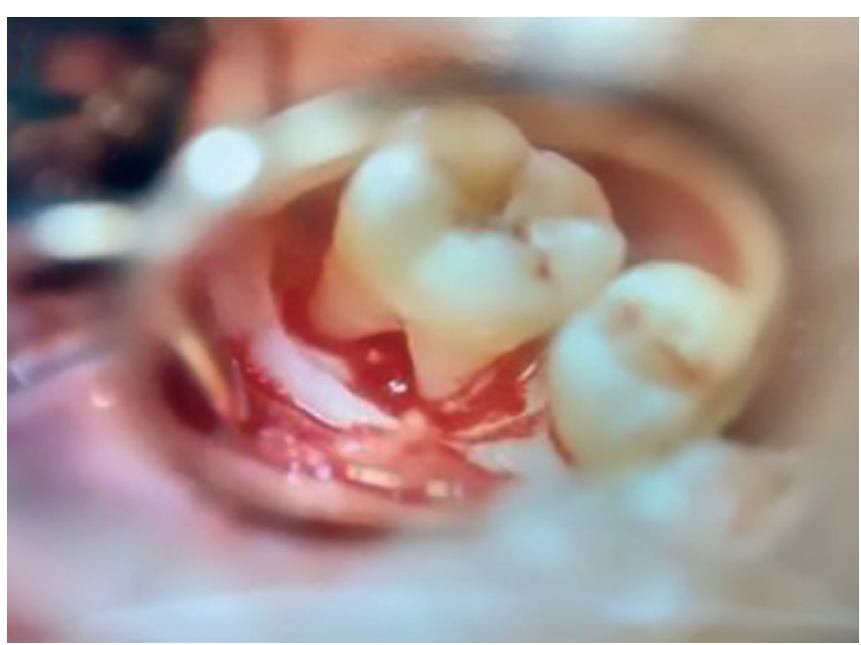

Fig. 7: Grade 2 furcation involvement of with flat marginal architechture. 


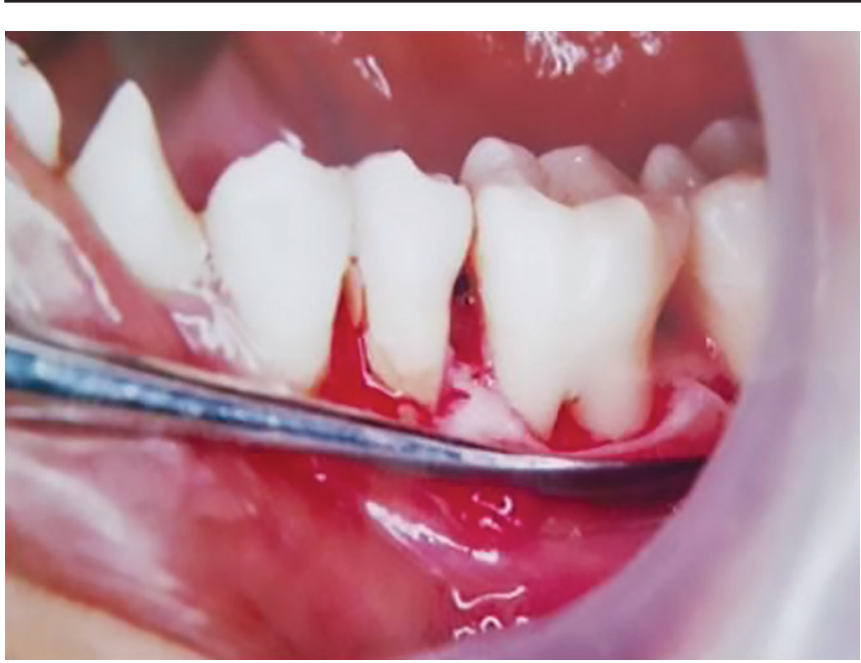

Fig. 8: Grade 2 furcation involvement

bone loss. In these defect base is present apical to the surrounding alveolar bone. These angular defects have accompanying infrabony pockets in most of the instances and these type of pockets usually have an angular defect underlying them. Further these angular or vertical defects are classified upon on presence of remaining osseous walls as three wall defects which are bordered by one tooth surface and three osseous surfaces, two wall defects (inter dental craters) which are bordered by two tooth surfaces and two bone or osseous surfaces (one facial and one lingual) and one wall defects which are bordered by two tooth surfaces, and bone surface (facial or oral) and soft tissue ${ }^{2}$ (Fig. 9).

\section{Hemisepta}

A vertical or angualar bone defect in the presence of adjacent roots such that only half of theinterdental septum is remained on one tooth is termed as hemiseptum.The remaining half of an inter-dental septum forms the proximal surface or wall of a one walled intrabony defect. ${ }^{2}$

The interdental septum is shared by two adjacent teeth. The hemisepta in relation to any tooth refines to the half of the septum adjacent to this specific tooth root is lost.

These one wall vertical defect occurring interdentally is called hemiseptum which can generally be seen on the radiograph but sometimes vertical defects which may be present on facial or buccal and lingual or palatal surfaces are not seen on radiographs and surgical exposures are the better way for determining the presence of defect and also the configuration of vertical bone defects. ${ }^{2}$

Osseous Craters: Craters are cup- or bowl-shaped alveolar defects in inter-alveolar bone with bone loss approximately equal on the contiguous roots or the concavities present in the crest of inter-dental alveolar bone and are

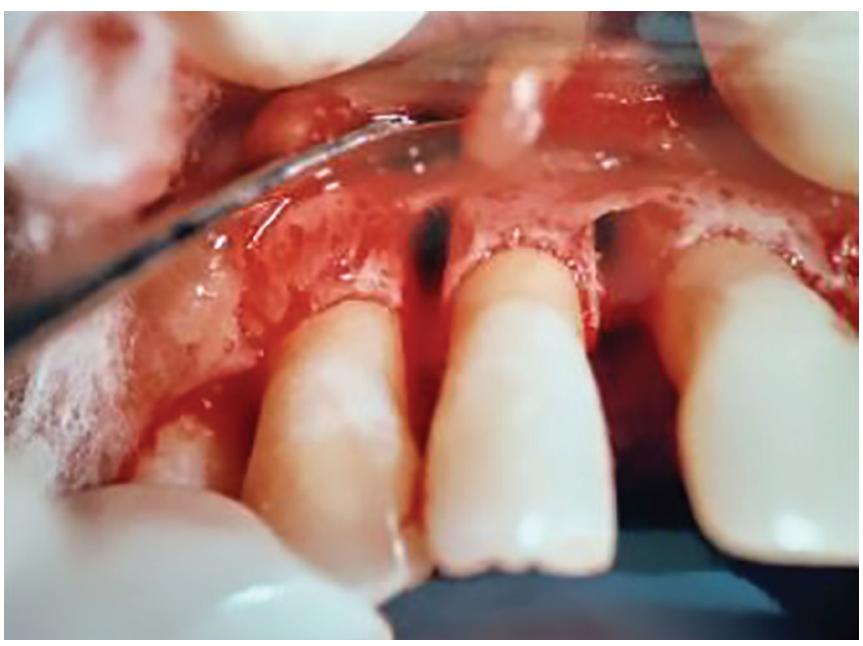

Fig. 9: Hemiseptum irt 23 (1 wall intrabony defect)

confined within the facial and lingual bony walls. In this type of defect the facial/buccal and lingual/ palatal walls are of unequal height. Craters are also classified by the number of osseous walls (i.e., a one-, two-, or threewalled) or combination bone defects also exist. ${ }^{14}$ These type of bone defects are frequently found about one third of all bone deformities or defects and about two thirds of mandibular bone defects and these defects are seen twice as common in posterior segments than in anterior segments. $^{20}$

The suggested reasons for such high prevalence of inter-dental craters are as follows: (i) as these areas show increased plaque accumulation as well as are difficult to clean; (ii) the flat or concave facio-lingual morphology of the interdental septum in mandibular molars may favor the formation of craters and the vascular patterns from gingiva to alveolar crest may also provide a pathway for extension of inflammation and formation of craters. ${ }^{20}$

Trench and Moat: When bone loss involves either two or three confluent surfaces of same tooth then the term rench is applied and when the bone loss involves all four surfaces of a tooth, then the term moat is applied. ${ }^{19}$

The periodontal treatment outcomes are assessed quantitatively based on defect angulations and depth defects in radiographs and there are certain classifications of defects radiographically given by Steffensen and Weber, ${ }^{21}$ Papapanou and Wennstrom, ${ }^{22}$ Cortellini and Tonetti ${ }^{23}$ and Tsitoura et al. ${ }^{24}$ as shown in the Table 2.

\section{Osseous Defects Model (Figs 10 and 11)}

To facilitate the descriptive visualization of various periodontal osseous defects in a mandible will serve as realistic demonstrable model. The true osseous defects morphology detection is possible only on surgical 


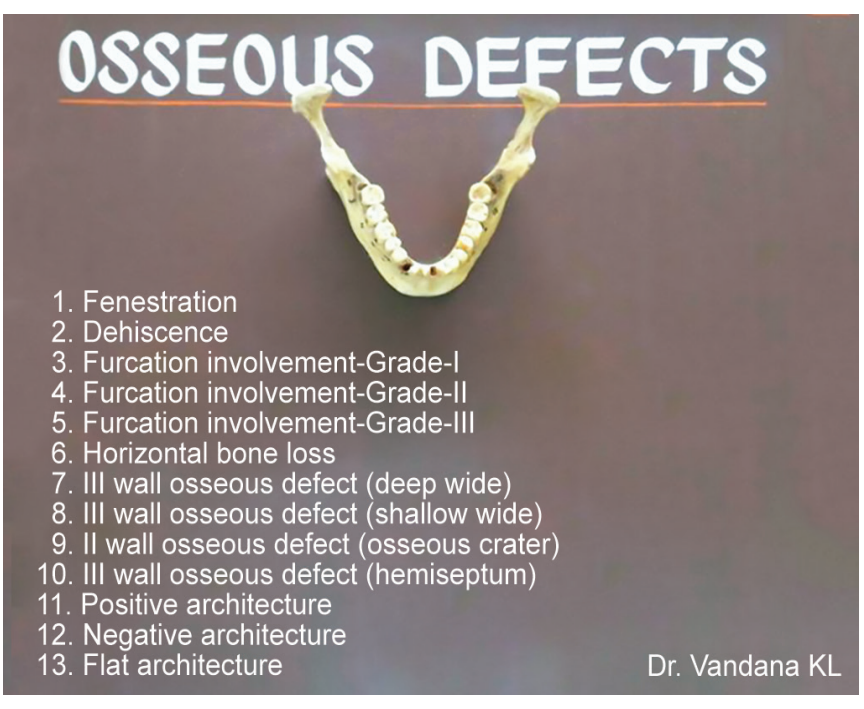

Fig. 10: Osseous defects model

exposure. The radiographic interpretation is often incomplete and the treatment protocol is decided only on surgical exposure. ${ }^{13}$

\section{How to identify, the Osseous Defects on Surgical Exposure}

- The periodontal osseous defects can be expressed separately as interproximal and buccal \lingual $\backslash$ palatal defects so that the treatment of osseous defects can be considered individually. In any given case occurrence of different types of defects are common.

- Interproximal periodontal osseous defects (IPOD)

- For any tooth root, the maximum number of osseous walls can be present are three.

- Identify the number of osseous walls in relation to periodontal defect of a particular tooth side.

Table 2: Radiographic classification of osseous defects

Steffensen and Weber ${ }^{21}$ classified based on radiographic intrabony defect angle as

a. Small angles $\left(0-45^{\circ}\right)$

b. Wide angles $\left(45-90^{\circ}\right)$.

Papapanou and Wennstrom ${ }^{22}$ classified depending on the depth of the defect in to

a. Degree 1-2 $\mathrm{mm}$

b. Degree 2-2.5 to $4 \mathrm{~mm}$

c. Degree $3->4.5 \mathrm{~mm}$

Cortellini and Tonetti ${ }^{23}$ classified based on radiographic defect angle in to

a. Narrow angle was defined as defect of $25^{\circ}$ or less

b. Wide angle was defined as defect of $37^{\circ}$ or more

Tsitoura et al. ${ }^{24}$ classified based on radiographic defect angle into
a. Narrow defect angle-Defect angle of $22^{\circ}$ or less
b. Intermediate defect angle-Defect angle between $22^{\circ}$ to $36^{\circ}$
c. Wide defect angle-Defect angle of $36^{\circ}$ or more

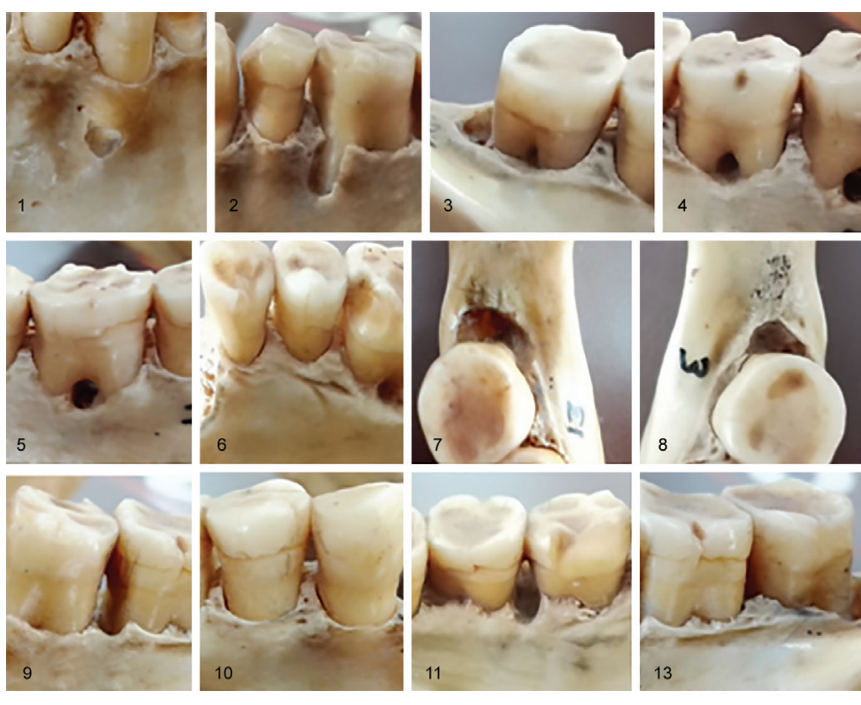

Fig. 11: Individual osseous defects

- The tooth root is always one of the walls of POD.

- Never try to name the interproximal wall as mesial or distal, however address it as interproximal wall. ${ }^{13}$

\section{To which Tooth the POD belongs to?}

The POD belongs to tooth where the root is exposed by pocket formation. Exception to this statement is two wall osseous crater where in buccal and lingual osseous walls are present where in the roots of both adjacent teeth are exposed. ${ }^{13}$

Crater: How to assess the levels of osseous walls: (Fig. 12)

To assess if both buccal and lingual walls of a crater are at same height or at different heights, a periodontal probe is kept horizontally touching both the alveolar crests, if it remains in same plane, the alveolar crests are at same levels and if not they are at different levels. This simple procedure will assist in the treatment protocol of craters with osseous wall at different levels. This step assists the visual examination of the clinician to confirm the bone levels of the crater. ${ }^{13}$

Combined defects: The apical part of combined defect will usually have higher number of alveolar bone walls than the total number of walls which are present coronally. The depth of the defect: Defect depth is measured from
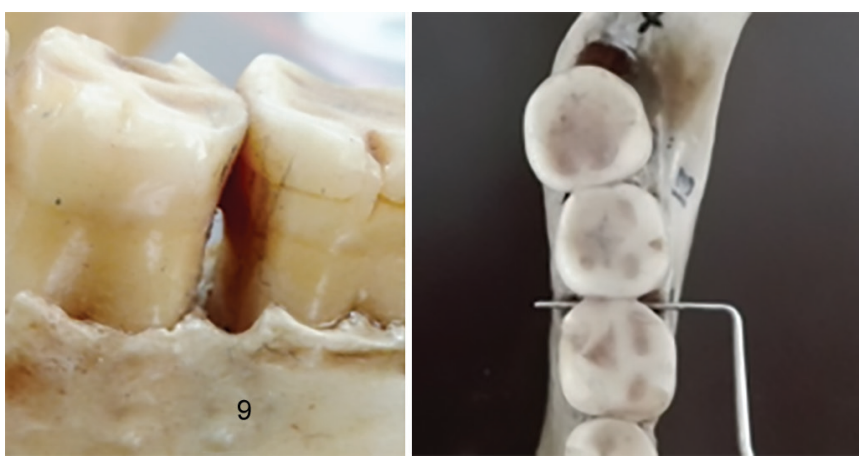

Fig. 12: How to assess crater 
alveolar crest to the base of the defect and CEJ to base of the defect.

\section{Clinical transfer of Proof of Delivery}

Periodontal osseous defects have to be interpreted as oral buccal/facial and interproximal defects as the treatment protocol has to be extended to both of them.

The physiologic architecture of the bone is responsible for contour and knife edge gingival margin and disease induced alterations of marginal bone influences the gingival morphology specially the bulbous bony contour.

In case of bulbous bony contour either developmental or acquired produces gingival enlargement clinically. The clinician has to ascertain whether the gingival enlargement per se by the transgingival probing method.

If it is gingival enlargement, the thickness will be increased. If it is due to bony enlargement the gingival enlargement remains same and the contributing factor as bulbous bony contour.

Identification and nomenclature of POD is confusing. Many a times there is no agreement amongst clinicians due to inconsistencies in classification and various overlapping terminologies. To some extent, the present paper provides amicable solution to clinicians to comprehended POD better. ${ }^{13}$

\section{CONCLUSION}

Periodontal osseous defects (POD) are an interesting part in periodontal destruction which was been never dealt to its finer aspects. A first attempt to comprehend the information and to expressit is the main objective of this review.

\section{REFERENCES}

1. Joseph P Fiorellini, David M Kim, Satoshi o Ishikawa. The tooth supporting structures. In: FA Carranza, editor. Clinical periodontology, 10th ed. Newdelhi: Elsivier publishers; 2007. pp. 68-92.

2. Fermin A Carranza and Henry H Takei. Bone loss and patterns of bone destruction. In: FA Carranza, editor. Clinical periodontology. 10th ed. Newdelhi: Elsivier publishers; 2007. pp. $452-66$.

3. Hausmann E, Allen K, Clerehugh V. What alveolar crest level on a bite-wing radiograph represents bone loss? J Periodontal 1991;62:570-572.

4. De Toledo BE, Barroso EM, Martins AT, Zuza EP. Prevalence of periodontal Bone Loss in Brazilian Adolescents through interproximal radiography. Int J Dent 2012:;2012:1-5.

5. Baljoon M, Natto S, Bergstorm J. Occurrence of vertical bone defects in dentally aware individuals. Acta Odontol Scand 2003;61:47-51.
6. Vrotsos JA, Parashis AO, Theofanatos GD, Smulow JB. Prevalence and distribution of bone defects in moderate and advanced adult periodontitis. J Clin Periodontol 1999;26: 44-48.

7. Kasaj A, Vasiliu Ch, Willershausen B. Assessment of alveolar bone loss and angular bony defects on panoramic radiographs. Eur J Med Res 2008;13:26-30.

8. Wu SK, Yeh HC, Chan CP. The prevalence and distribution of bone defects in patients with moderate to advanced periodontitis. Chang Gung Med J 2001;24:423-430.

9. Goldman HM, Cohen WD. The Infrabony Pocket; classification and treatment. J Periodontol 1958;29:272-291.

10. Pritchard J. The infrabony pocket classification. J Advanced Periodontal Disease. 2nd ed. W. B. Saunders, Philadelphia. 1972; 558-565.

11. Clarke M, Bueltman K. Anatomic considerations in periodontal surgery. J Periodontol 1971;42:610-625.

12. Papapanou PN, Tonetti MS. Diagnosis and epidemiology of periodontal osseouslesions. Periodontol 2000;22:8-21.

13. Vandana KL, Bharath Chandra GNR, Sadanand K. Classification of Periodontal Osseous Defects. In: Vandana KL, editor. Periodontal osseous defects an Insight, 1st ed. Republic of Maldova: Lambert academic publishers; 2017. p. 8-9.

14. Manson JD, Nicholson $\mathrm{K}$. The distribution of bone defects in chronic periodontitis. J Periodontol 1974;45:2:88-92.

15. Nery EB, Corn H, Eisenstein IL. Palatal exostoses in the molar region. J Periodontol 1977;48:663.

16. Nimigean VR, Nimigean V, Bencze MA, Dimcevici-Poesina N, Cergan R, Moraru S. Alveolar bone dehiscences and fenestrations: an anatomical study and review. Rom J Morphol Embryol 2009;50:391-397.

17. Glickman I, Smulow J. Buttressing bone formation in the periodontium, J Periodontol 1965 Sep;36(5):365-70.

18. Larato DC. Some anatomical factors related to furcation involvements. J Periodontol 1965;46(10):608.

19. Karn KW, Shockett HP, Moffitt WC, Gray JL. Topographic classification of deformities of the alveolar process. J Periodontol 1984;55:336-340.

20. Saari JT, Hurt WC, Briggs NL. Periodontal bony defects on the dry skull. J Periodontol 1968;39:278.

21. Steffensen B, Weber HP. Relationship between the radiographic periodontal defect angle and healing after treatment. J Periodontol 1989;60:248-254.

22. Papapanou PN, Wennstrom JL. The angular bony defect as indicator of future alveolar bone loss. J Clin Periodontol 1991; 18:317-322.

23. Cortellini P, Tonetti M. Radiographic defect angle influences the outcomes of GTR therapy in intrabony defects. 77th general Session of the IADR, Vancouver,Canada, March 1999:10-13.

24. Tsitoura E, Tucker R, Suvan J, Laurell L, Cortellini P, Tonetti M. Baseline radiographic defect angle of the intrabony defect as a prognostic indicator in regenerative periodontal surgery with enamel matrix derivative. J Clin Periodontol 2004;31:643-647. 